\title{
An autonomous photovoltaic system sizing program for office applications in Africa
}

\author{
Bert Herteleer ${ }^{1}$, Jan Cappelle ${ }^{2}$, Johan Driesen ${ }^{3}$ \\ ${ }^{1}$ Catholic University College Ghent, Gebroeders De Smetstraat 1, B-9000 Gent, Belgium - bert.herteleer@kahosl.be \\ ${ }^{2}$ Catholic University College Ghent, Gebroeders De Smetstraat 1, B-9000 Gent, Belgium - jan.cappelle@kahosl.be \\ ${ }^{3}$ Katholieke Universiteit Leuven, ESAT-ELECTA, Kasteelpark Arenberg 10 bus 2445, B-3001 Heverlee, Belgium - johan.driesen@esat.kuleuven.be
}

\begin{abstract}
A spreadsheet tool and underlying model was developed to aid non-expert users in sizing off- or on-grid photovoltaic systems with battery back-up for office applications in Africa. The tool offers the user a number of choices which help in the decision process. The model is based on the concept of energy equivalence and extended by taking into account nonideal behaviours of photovoltaic system components, modelled as efficiency deviations. The spreadsheet tool uses freely available data, such as PV system component manufacturer's data sheets, as well as climatic data from the NASA SSE database. The irradiation data on the plane-of-array is calculated for the chosen location using the well established HDKR irradiation model. The obtained irradiation and energy output were compared to free services from Soda-is and the HOMER sizing software. The predicted energy output comparison of identical off-grid photovoltaic systems per location for two sites in Africa were within $14 \%$ of the value given by HOMER. A "performance ratio"-equivalent efficiency was calculated as part of the model. This intuitive approach to photovoltaic system sizing reduces the learning curve for non-expert users. The predicted "performance ratio"-equivalent efficiency can be used as a predictive analytic tool for grid-connected and off-grid photovoltaic systems.

Keywords: photovoltaic system sizing, efficiencies,

energy equivalence, performance ratio
\end{abstract}

\section{Introduction}

This paper outlines the main points of an off-grid photovoltaic (PV) system sizing spreadsheet tool and its underlying model. It was designed for companies with offices located in Africa which suffer from an unreliable or non-existent power supply from the grid. There is a large range of possible photovoltaic system sizing programs available, both paid and free. Well-known and much-used PV system sizing programs are PVSyst[1] and HOMER[2]. Typically, these programs have a long and steep learning curve.

The spreadsheet tool described in this paper was configured to be easy and fast to use by non-experts and be based on freely available data on the internet.

An important part of this tool is its reliance on photovoltaic module and inverter manufacturer's data sheets to predict
PV system behaviour. In this way, less experience is required by users to perform a system sizing.

A number of user-friendly measures were implemented, such as providing prices of available PV system components, as well as basic financial decision-aiding criteria, such as the expected payback time of the chosen system.

As with other PV system sizing tools, a minimum amount of inputs and design choices must be given by the user to achieve meaningful system sizing results. A short enumeration of the required inputs for the tool is given:

1. Daily $\left(E_{r e q, d}\right)$ or yearly $\left(E_{r e q, y}\right)$ energy use, current or projected for the office in question.

2. Location parameters: latitude $(\phi)$, longitude $(\lambda)$ as well as physical installation parameters: tilt angle $(\beta)$ and azimuth angle $(\gamma)$.

3. Long-term daily values of the irradiation on a horizontal surface $\left(G_{d}\right)$ and the maximum daily temperature at $10 \mathrm{~m}$ above the surface of the location $\left(T_{\max }\right)$. These are obtained as daily averaged data [3] from the NASA SSE database for a 22-year timespan.

4. Photovoltaic (PV) system on- or off-grid components (a reduced list of parameters is given below):

- PV modules: efficiencies $\left(\eta_{P V}\right)$, power temperature coefficient $\alpha$, module power $\left(W_{p}\right.$ or $W$ ), module area $A_{P V}$

- Inverters: Weighted European efficiency $\eta_{I n v, E u}$, maximum DC and AC rated power $\left(P_{D C, \max }\right.$, $\left.P_{A C, \text { nom }}\right)$, power rating.

- Bidirectional inverters: AC-DC and DC$\mathrm{AC}$ conversion efficiencies $\left(\eta_{A C D C, E u} \&\right.$ $\left.\eta_{D C A C, E U}\right)$, as well as the energy capacity of the bidirectional inverter $E_{\text {Inv,Bidir }}$

- Batteries: Voltage level $V_{b a t t}$ and discharging capacity $C_{b a t t}(\mathrm{Ah})$, as well as typical efficiency $\eta_{\text {batt }}$

5. Lifetime of PV system use and other financial parameters, such as the required Return on investment (ROI), as chosen by the user. 
The most important choices and actions that the user must do are:

- Choose between detailed daily energy consumption OR yearly consumption from which a daily average value is computed.

- Copy the correct long-term (22 years) daily temperature and irradiation values from the NASA SSE database [3].

- Choose the PV system components from the available database in spreadsheet OR personal input according to the template, based upon data sheets from a different manufacturer.

- Choosing which financial and practical considerations are most important for PV system sizing (e.g. amount of days of autonomy, if required, available roof area for PV modules, required Return on Investment (ROI), ... )
- Choosing the PV system type: detailed daily analysis of on/off-grid PV system or a more generic averaged on/off-grid PV system sizing.

This leads to the following output:

1. The most important result is a calculated PV onor off-grid system (depending on user choice) which satisfies the input yearly electrical energy requirement. Investment cost of system is also part of the output, as well as simple financial analysis (ROI, payback period). In this fashion, users can make an informed choice on the criterion that is most important according to personal preferences.

2. A Performance Ratio (PR)-equivalent efficiency value is computed, which can be used as a diagnostic tool to verify the correct functioning of the PV system, once it has been installed.

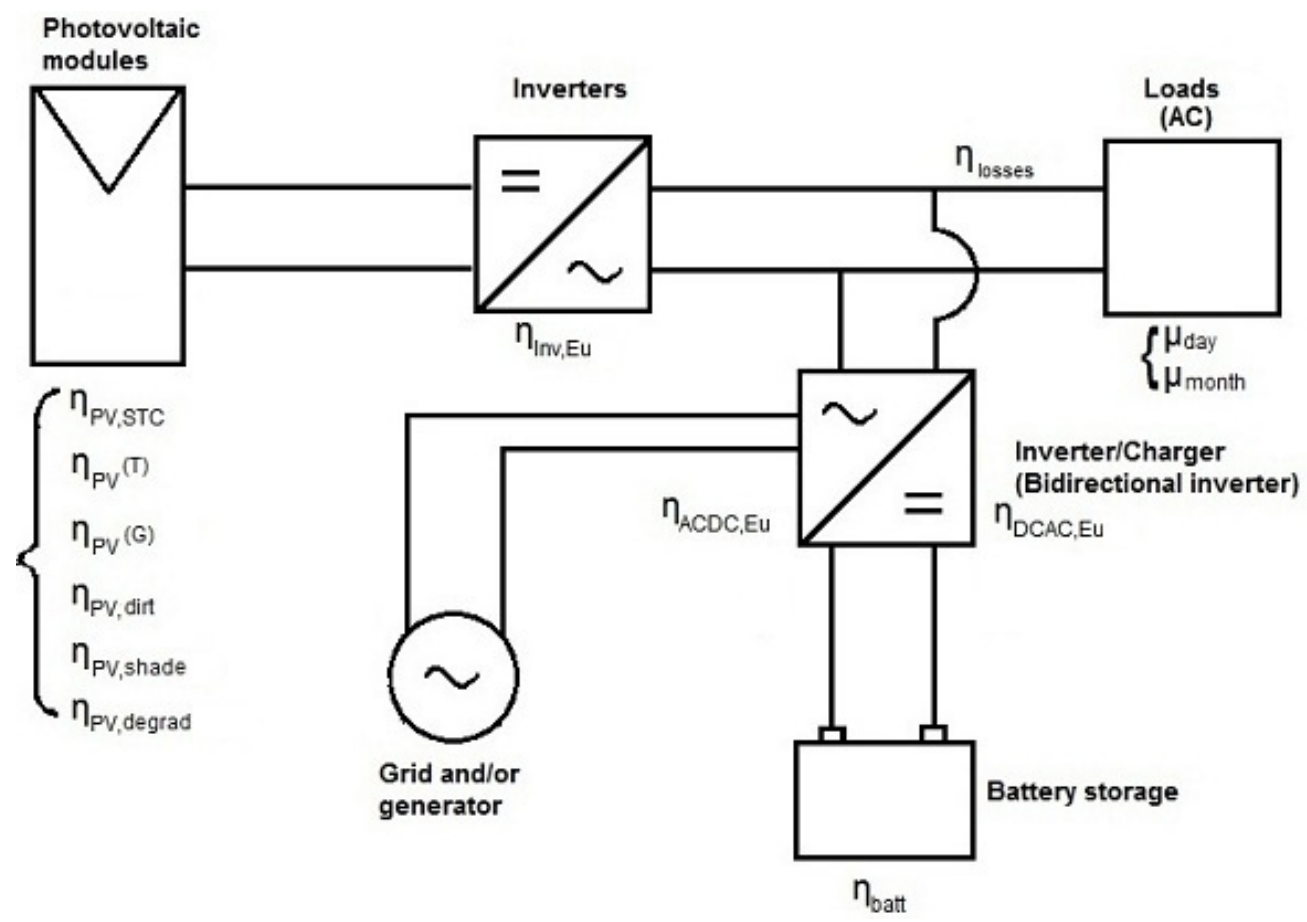

Figure 1: Schematic for off-grid or back-up PV system model

\section{Underlying model}

The model upon which the tool depends is based on the premise of energy equivalence (electrical energy consumed must be delivered by PV "generator") as described in literature by [4], [5] and others. This concept is extended by taking into account that systems are non-ideal and have an inherent efficiency, an approach similar to [6]. Taking standard testing conditions (STC) as the reference normalizing value for "ideal"/reference PV systems, welldocumented losses (such as the PV module temperature dependence) are modelled as efficiency losses. To predict the behaviour of PV systems with as much accuracy as possible, three main areas are modelled:

1. The required amount of electrical energy per day. To better account for a possible imprecise estimation of the electrical energy requirements by non-expert users, two load-modifying factors are used: $\mu_{d}$ and $\mu_{m}$. (see section 2.1

2. The amount of solar energy which will be received by PV modules with a certain tilt angle $\beta$ and azimuth angle $\gamma$ at a certain latitude $\phi$ and longitude $\lambda$ on earth: the daily plane-of-array irradiation $G_{d}(\beta, \gamma)$ must be predicted with a sufficient degree of certainty and accuracy. For this purpose, the HDKR-model described by [7] is used, as it is robust, well established within the PV system sizing industry and its relative ease of implementation in spreadsheet software.

3. The behaviour of PV system components (PV modules, inverters, batteries, bidirectional inverters) and their influence on the total system efficiency must be taken into account. This is done by modelling most well-established PV system losses as proportional efficiency losses. 
The autonomous AC-coupled PV system model with battery back-up is shown in figure 1 . Its on-grid equivalent is obtained by removing the bidirectional inverter and batteries from the diagram. Using the topology of figure 1 . the efficiency deviations from STC as well as other losses can be explicitly incorporated to calculate the total system efficiency $\eta_{\text {syst,aut }}$ for an autonomous PV system. Its ongrid equivalent value $\eta_{\text {syst,grid }}$ is found by assuming that no energy needs to be stored in the batteries, which leads to the exclusion of the bidirectional inverter and battery efficiencies.

\subsection{PV system efficiencies and energy equivalence}

By taking the standard testing conditions as specified in data sheets of PV modules and inverters and introducing efficiency differences, an intuitive understanding of PV systems and their sizing can be obtained. This eases the learning process for non-expert users of the sizing tool in a significant way.

The start for the efficiencies approach is by following the path of electrical energy from the PV module for a grid-connected PV system without battery back-up. In its general form this is a grid-connected PV system efficiency:

$$
\eta_{\text {syst,grid }}=\eta_{P V, \text { tot }} \cdot \eta_{\text {Inv }, \text { Euro }} \cdot \eta_{\text {losses }}[\%]
$$

The value of $\eta_{\text {Inv,Euro }}$ is specified by inverter manufacturers on the data sheet of the chosen inverter. ${ }^{1}$ Losses which are design-specific, such as for example, cable losses for varying lengths and sections, are gathered in the value $\eta_{\text {losses }}$. The total efficiency $\eta_{P V \text {,tot }}$ associated with PV modules can be calculated as:

$\eta_{P V, \text { tot }}=\eta_{P V}(T) \cdot \eta_{P V}(G) \cdot \eta_{P V, \text { shade }} \cdot \eta_{P V, \text { dirt }} \cdot \eta_{P V, \text { degrad }}$

The respective items in equation 2 are calculated as:

$$
\begin{aligned}
\eta_{P V}(T) & =1+\alpha \cdot\left(T_{\text {cell }}-25^{\circ} C\right) \\
\eta_{P V}(G) & =1+\kappa \cdot \ln \left(\frac{G}{G_{S T C}}\right) \\
\eta_{P V, \text { shade }} & =\text { estimated from local conditions } \\
\eta_{P V, \text { dirt }} & =\text { estimated from local conditions } \\
\eta_{P V, \text { degrad }} & =1-\frac{\Delta P_{P V}(\text { life }) \%}{P V \text { life }} \cdot \frac{\text { Est.use time }}{2} \\
& =1-\frac{20 \%}{25 \text { years }} \cdot \frac{\text { \#years of use }}{2}
\end{aligned}
$$

Eq. 3 is the normalized version of the temperature dependence equation of PV module power[5], compared to STC. Eq. 4 is an empirical formula which indicates the irradiance-dependence of PV modules. The value of $\kappa$ is technology-dependent: from a study[8] it was assigned $4 \%$ for crystalline $\mathrm{Si}$ and $1.5 \%$ for thin-film technologies. Eq. 7 assumes a linear degradation in PV power per year. The estimated time of PV system use is divided by 2 to obtain the degradation-dependent efficiency of PV modules halfway through their estimated useful lifetime. Depending on the intended use, this can be 25 years, whilst other companies might envision a much shorter period of use, e.g. 5 years. Eq. 1 is then extended and modified by taking into account the conversion efficiencies required for battery back-up. The DC electrical energy delivered by PV modules working in ideal STC conditions, is partly lost through several conversion steps, described by their relative efficiencies. A portion of the energy that is generated by the PV modules will have to be stored in the batteries. Introducing the daily solar-load matching factor $\mu_{d}$, a better approximation of the real energy use at an office can be done, as the consumption of electrical energy is often underestimated. It is calculated and defined as:

$$
\mu_{d}=\frac{E_{r e q, \text { hours }}}{E_{\text {req }, d}}\left[\frac{k W h / d a y}{k W h / d a y}=\%\right]
$$

It is thus the ratio of energy required during the designated (office) hours to the total energy required during the day. It is assumed that office hours coincide with the availability of sunlight. As such, the value " $1-\mu_{d}$ " describes the portion of the total daily electrical energy requirement that must be stored in batteries. This energy will have to be generated by PV modules at a different moment in time, which logically leads to a larger PV array requirement.

The storage of the electrical energy leads to the following analysis: The AC electrical energy which is delivered by the inverters after the PV modules must be converted to DC $\left(\eta_{A C D C, E u}\right)$ to be stored in the batteries which have a general average efficiency $\eta_{b a t t}$. Once the electrical energy is required from the batteries, it must be converted back into AC electrical energy $\left(\eta_{D C A C, E u}\right)$. The autonomous PV system with back-up efficiency is then calculated as a modification of Eq. 11 using an autonomous PV system modifier $\eta_{\text {aut }, \text { modif }}$ :

$\eta_{\text {aut }, \text { modif }}=\mu_{d}+\left(1-\mu_{d}\right) \cdot \eta_{A C D C, E u} \cdot \eta_{b a t t} \cdot \eta_{D C A C, E u}$

which leads to

$$
\eta_{\text {syst,aut }}=\eta_{\text {syst, }, \text { rid }} \cdot \eta_{\text {aut }, \text { modif }}
$$

While current "intelligent" battery-inverters or bidirectional inverters do not specify a weighted European efficiency such as their PV module counterparts, an effort was made to recalculate the specified maximum efficiency to a weighted efficiency. As leading manufacturers do not yet detail differences in conversion efficiency from $\mathrm{AC}$ to DC $\left(\eta_{A C D C, E u}\right)$ electrical energy and DC-AC $\left(\eta_{D C A C, E u}\right)$, these efficiency values are estimated to be equal in value.

\subsection{Photovoltaic array sizing}

The elementary calculation of the required PV module power, which uses the amount of Peak Solar Hours $\tau_{d}(\beta, \gamma)$ per day which reach PV modules with a certain tilt angle $\beta$ and azimuth angle $\gamma$ is given as:

$$
P_{P V}=\frac{E_{r e q, d}}{\tau_{d}(\beta, \gamma)}
$$

\footnotetext{
${ }^{1}$ The system designer can opt to choose to use $\eta_{I n v, C E C}$ from the California Energy Commission for high irradiation conditions. Not all inverter manufacturers give this value yet.
} 
Taking into account that electrical energy consumption is typically higher in winter than summer months, (excepting air conditioning use in very hot climates) a standardized monthly consumption-matching factor $\mu_{m}$ can be introduced. The factor $\mu_{m}$ indicates the degree of deviation by the monthly averaged electrical energy consumption, compared to the annual mean of electrical energy consumption. It is calculated as:

$$
\mu_{m}=\frac{\sum_{i=1}^{i \leq 31}\left(E_{\text {req, tot }, d}\right)_{i}}{E_{\text {req, tot }, \text { year }}} \div \frac{\text { Days per month }}{\text { Days per year }}[\%]
$$

If the highest electrical energy consumption occurs during months with the lowest irradiation, this value will prevent a PV system to be undersized. By dividing the value of $\tau_{d}(\beta, \gamma)$ by $\mu_{m}$ for all months of the year, the worst irradiation-consumption month-combination of the year can be found, with which an off-grid system will be sized:

$$
\left(\frac{\tau_{d}(\beta, \gamma)}{\mu_{m}}\right)_{\text {min }}=\left.M I N\right|_{i=J a n} ^{D e c}\left(\frac{\tau_{d}(\beta, \gamma)_{i}}{\mu_{m, i}}\right)
$$

By extending Eq. 11 using $\eta_{\text {syst,aut }}$ and the loadmodifying values $\mu_{d}$ and $\mu_{m}$, the PV module power is modified to better predict and deal with eventual non-ideal/standard working conditions, thus reducing the possibilities of power outages during (prolonged) low lighting conditions:

$$
P_{P V, \text { aut }}=\frac{E_{\text {req, }}}{\left(\frac{\tau_{d}(\beta, \gamma)}{\mu_{m}}\right)_{\min } \cdot \eta_{\text {syst }, \text { aut }}}
$$

For grid-connected systems (assuming a stable and dependable electrical grid), Eq. 14 can be modified by setting $\mu_{d}=100 \%$ which makes $\eta_{\text {aut,modif }}$ equal to unity (see Eq. 9), and taking the yearly average value $\left(\frac{\tau_{d}(\beta, \gamma)}{\mu_{m}}\right)_{a v g}$ instead of the worst irradiation-consumption month-combination of the year. Thus:

$$
P_{P V, \text { grid }}=\frac{E_{r e q, d}}{\left(\frac{\tau_{d}(\beta, \gamma)}{\mu_{m}}\right)_{\text {avg }} \cdot \eta_{\text {syst }, \text { grid }}}
$$

\subsection{Inverter sizing}

Inverters are sized according to their intended use (one or three phase operation, ...). The simplest method is to take the PV module peak power $\left(k W_{p}\right)$ and then choose one or more inverters that approximate this value by their $\mathrm{AC}$ nominal power. E.g:

$$
N_{\text {Inv }}=\frac{P_{P V}}{P_{\text {Inv }, A C}} \quad\left[\frac{k W_{p}}{\frac{k W_{A C}}{\text { Inverter }}}=\# \text { Inv }\right]
$$

Manufacturers of PV module inverters then supply a power ratio criterion:

$$
\text { Power ratio }=\frac{P_{D C, \text { inv }, \text { max }}}{P_{P V}}\left[\frac{k W_{I n v, D C}}{k W_{p}}=\%\right]
$$

The values of the power ratio are then preferably

$90 \leq$ power ratio $\leq 110 \%$ or $80 \leq$ power ratio $\leq 120 \%$, depending on the manufacturer. The larger range $(80 \%$ to $120 \%$ ) is used in the spreadsheet tool.

\subsection{Battery sizing}

While methods for battery sizing for off-grid systems are well documented as in e.g. [9], [10], the chosen equation is indicated here for clarity, with $N_{a u t}$ the number of days of autonomy and $D O D_{\max }$ the maximum depth-of-discharge (DOD) of the batteries [9]:

$$
E_{\text {batt }, \text { tot }}=\frac{N_{a u t} \cdot E_{\text {req, }}}{D O D_{\max }}
$$

The number of batteries $N_{b a t t}$ can then be calculated :

$$
N_{\text {batt }}=\frac{E_{\text {batt }, \text { tot }}}{E_{\text {batt }}}\left[\frac{k W h}{\frac{k W h}{\text { battery }}}=\text { batteries }\right]
$$

\subsection{Bidirectional inverter sizing}

Bidirectional battery inverters have several limitations such as the peak power that they can convert from AC to DC or vice versa. The amount of stored energy that a bidirectional inverter can manage is given by the following empirical equation:

$$
\begin{gathered}
E_{I n v, \text { Bidir }}=\left(\frac{C_{b a t t, \max }-C_{b a t t, \text { min }}}{2}\right) \cdot V_{\text {batt }} \cdot M_{S F} \\
{[V \cdot k A h=k W h]}
\end{gathered}
$$

Wherein $C_{b a t t}$ is the $\mathrm{C} 10$ discharging rate of batteries in $\mathrm{kAh}, V_{\text {batt }}$ the voltage of the battery bus and the value $M_{S F}$ is a manufacturer's sizing factor. For example, with SMA's Sunny Island SI5048 ${ }^{2}$, this value is $\approx 5.9$, from calculations based on examples given in [11]. The choice of the DC working voltage of the bidirectional inverter will influence the final amount of required batteries. If necessary, a number of iterative steps must be taken to coordinate the number batteries to the necessary amount of bidirectional inverters. The number of bidirectional inverters required can then be calculated as:

$$
N_{\text {Inv }, \text { Bidir }}=\frac{N_{\text {batt }} \cdot E_{\text {batt }}}{E_{\text {Inv }, \text { Bidir }}}\left[\frac{\# \cdot k W h}{k W h}=\#\right]
$$

\subsection{Irradiation and temperature data}

An average climatic year was constructed by averaging daily irradiation values of every calendar day from the 22 years of available data in the NASA SSE database for daily averaged data. For example, the irradiation value for the average climatic year for "January $1 \mathrm{st}$ " is calculated by averaging the irradiation values for the 22 occurrences in the database. The same calculation is applied to the remainder of the average climatic year.

Additionally, the same process is repeated for the Maximum Air Temperature at $10 \mathrm{~m}$ above the surface of the location input into the NASA SSE database.

\footnotetext{
${ }^{2}$ No specific preference for this manufacturer is implied.
} 


\section{Implementation of model}

A sizing tool based upon the model described above was implemented in spreadsheet software. The value of $E_{r e q, d}$ was calculated from annual values which must be input by the user.

The generated electrical energy over one year is then calculated as a sum of the generated energy per day by the PV system. The generated energy is at least equal to the required electrical energy consumption over the course of a year.

User-friendly measures which were implemented in the spreadsheet tool:

- A small database of main PV system components (PV modules, inverters, bidirectional inverters and batteries) with respective prices found online is available to the user. This way, the estimated system cost can be calculated for a chosen PV system configuration.

- The user can input the expected lifetime of use of the PV system. Using this value, the payback period and Net Present Value and expected Return On Investment are calculated, offering the user a range of financial criteria for a $\mathrm{GO} / \mathrm{NO} \mathrm{GO}$ decision.

- A number of PV system configurations based on knowledge of expected electrical energy consumption allows the user to perform a rapid system sizing and cost estimation. These configurations are based on whether detailed electrical energy consumption information is available, as well as financial and space constraints. In this context, critical appliances were defined if the user would input detailed electrical energy consumption:

A critical appliance is defined as one that is of vital importance to the efficient working of an office. Typical examples would be PCs and lighting, while vacuum cleaners or coffee machines could be chosen to be noncritical.

This allows the user to design and choose a PV system back-up configuration which best matches the expected needs. The possibilities as given in the spreadsheet tool are:

1. Annual electrical energy consumption input. Ongrid PV system (PV modules + inverters) for the full office is sized.

2. Annual consumption is known. Off-grid PV system with battery back-up for the full office (PV modules, inverters, batteries and bidirectional inverters)

3. Detailed electrical energy consumption input: Off- grid system: full office (PV modules), full office back-up.

4. Detailed consumption input: Off-grid system: full office (PV), critical appliances back-up.

5. Detailed input: On-grid system: full office (PV).

6. Detailed input: Off-grid system: critical appliances (PV) and critical appliances back-up.

7. Detailed input: On-grid system: critical appliances (PV) only.

This way, a user can make a decision based on facts to ask an installer or consulting firm to perform a more detailed definitive system sizing.

An important aspect of this work is the scalability of predicted losses in a PV system. While the Performance Ratio (PR) is a useful parameter in identifying how well an installation works, it is defined using measured absolute annual final $\left(Y_{y, f}\right)$ and reference $\left(Y_{y, r}\right)$ yields[12]:

$$
P R=\frac{Y_{\text {period,f } f}}{Y_{\text {period }, r}} \stackrel{\text { typically }}{=} \frac{Y_{y, f}}{Y_{y, r}}\left[\frac{k W h / y r}{k W h / y r}=\%\right]
$$

The advantage of using this model is that a PRpredicting calculation is done when calculating $\eta_{\text {syst,grid }}$ (Eq. 11) for grid-connected systems, while $\eta_{\text {syst,aut }}$ (Eq. 10) will give an approximation of a PR-like value for autonomous systems. Here, further work to incorporate the usage factor[13] for off-grid systems as a system metric can be done.

\section{Results}

As correct irradiation values are very important for PV system sizing, an analysis of predicted annual irradiation on a tilted, oriented plane was done. It compared spreadsheet values to the Soda-is solar radiation database for 50 sites along a North-South axis. The values showed a mean difference of $-3 \%$ with standard deviation of $7.6 \%$. The output of both methods can be seen in figure 2. This comparison was between the long-term average (spreadsheet, NASA SSE data) versus a single year (Soda-is), which shows a strong similarity to interannual variability values reported by [14], albeit in part over a different geographical region.

The spreadsheet tool was compared to HOMER by performing an off-grid system sizing for two locations in Africa. [8] In these cases, the predicted irradiation by the tool versus HOMER differed by $-0.5 \%$ and $+8 \%$. The respective annual electrical energy output (with output from the spreadsheet used as input for the HOMER software) was $-14 \%$ and $+6.5 \%$. The difference in values are comparable in range to results found by [15], [16]. 


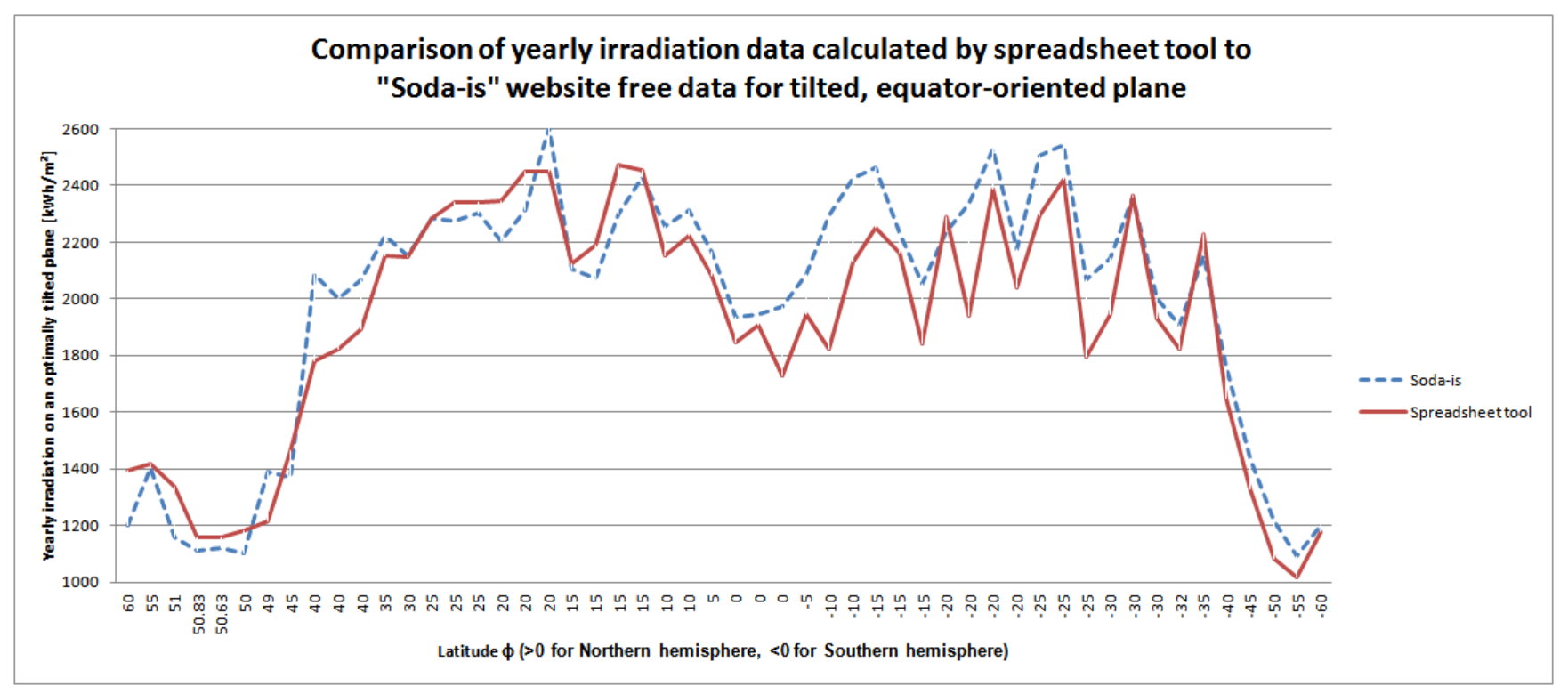

Figure 2: Yearly irradiation comparison for a tilted, equator-oriented plane between "Soda-is" data versus a climatic average year irradiation calculated in spreadsheet software for different locations on Earth. Values between $-30^{\circ}$ and $+30^{\circ}$ of latitude are mostly in Africa.

\section{Conclusions}

This paper presents an intuitive tool and model for photovoltaic system sizing, both grid-connected and offgrid with battery back-up. The spreadsheet tool allows for rapid system sizing with a shorter and easier learning curve for non-expert users. The focus of the tool is for offices located in Africa which suffer from an unreliable electrical energy supply.

While the tool was designed with Africa in mind, it can be easily employed in any country within the "sun belt" of the world, as the NASA SSE database and the spreadsheet tool are not limited to Africa.

The performance ratio can be predicted before system installation, allowing a comparison between modelled and measured data. The modularity and scalability of the model can be easily adapted to audiences interested in solar power, giving a possible range of detail to be chosen depending on the intended use.

\section{Note \& acknowledgement}

The tool and its instruction manual are free to use; a request to the authors for the tool can be done via email.

The research is funded by a Ph.D. grant of the Agency for Innovation by Science and Technology (IWT).

\section{References}

[1] Geneva University, “User's Guide: PVsyst 5 Contextual Help."

[2] HOMER Energy LLC, "HOMER: Hybrid Optimization Model for Electric Renewables, v. 2.68."

[3] J. M. Kusterer, "NASA Surface meteorology and Solar Energy: Daily Averaged Data," Available at http://eosweb. larc.nasa.gov/cgi-bin/sse/daily.cgi?email=

[4] L. Castañer, S. Bermejo, T. Markvart and K. Fragaki, "Energy Production by a PV Array," in Practical Handbook of Photovoltaics: Fundamentals and Applications, T. Markvart and L. Castañer, Eds. Elsevier Advanced Technology, 2003.

[5] T. Markvart, Ed., Solar Electricity, 2nd ed. John Wiley \& Sons, 2000.

[6] M. Ibrahim, "Straight forward technique for sizing standalone PV hybrid systems," in 20th - EU-PVSEC, June 2005.

[7] J. A. Duffie, W. A. Beckman, Solar Engineering of Thermal Processes, Second ed. Wiley-Interscience, 1991.

[8] B. Herteleer, "Berekenen en dimensioneren van autonome fotovoltaïsche installaties voor kantoren in Afrika [Calculating and sizing of autonomous photovoltaic systems for offices in Africa]," Master's thesis, Catholic University College Ghent, 2011.

[9] D. Spiers, "Batteries in PV Systems," in Practical Handbook of Photovoltaics: Fundamentals and Applications, T. Markvart and L. Castañer, Eds. Elsevier Advanced Technology, 2003.

[10] D. Gesellshaft fur Sonnenenergie (DGS LV Berlin BRB), Planning and Installing Photovoltaic Systems: A Guide for Installers, Architects and Engineers, 2nd ed. Earthscan, 2008.

[11] SMA Solar Technology, "Sunny Island System Guide."

[12] H. Haeberlin and C. Beutler, "Normalized Representation of Energy and Power for Analysis of Performance and On-line Error Detection in PV-Systems," in 13th EU-PVSEC.

[13] U. Jahn, B. Grimmig and W. Nasse, "Analysis of Photovoltaic Systems," Available at http://www. iea-pvps-task2.org/public/download/Report_IEA_PVPS_ T2_01_2000f.pdf

[14] M. Š́ri, T. Huld, E. D. Dunlop, M. Albuisson, M. Lefèvre, L. Wald, "Uncertainties in photovoltaic electricity yield prediction from fluctuation of solar radiation," in 22nd EUPVSEC, Milan, Italy, 2007.

[15] J. S. Stein, "Results of Model Intercomparison - Predicted vs. Measured System Performance," May 2011.

[16] C. P. Cameron, J. S. Stein, C. A. Tasca, "PV Performance Modeling Workshop Summary Report,” May 2011. 